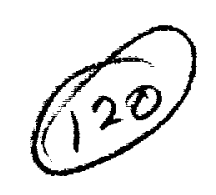

\title{
0216
}

\section{THERMOCAPILLARY FLOW AND AGGREGATION OF BUBBLES ON A SOLID WALL}

Hiroki Kasumi, Yuri E. Solomentsev', Scott A. Guelcher ${ }^{2}$, John L. Anderson and Paul J. Sides

Carnegie Mellon University, Department of Chemical Engineering, Pittsburgh, PA 15213, ps7r@andrew.cmu.edu, ${ }^{1}$ Motorola, Austin, TX 78721,

${ }^{2}$ Bayer Corporation, South Charleston, WV 25303

\section{ABSTRACT}

During the electrolytic evolution of oxygen bubbles forming on a vertically oriented transparent tin oxide electrode, bubbles were found to be mutually attractive $[1,2]$. The mechanism of the aggregation had never been explained satisfactorily until Guelcher et al. [3] attributed it to thermocapillary flow. The gradient of surface tension of the liquid at the bubble's surface, which was established because of reaction heat and ohmic heat loss at the electrode wall, drives flow of the liquid adjacent to each bubble; the bubble "pumps" fluid along its surface away from the wall. Fluid flows toward the bubble to conserve mass and entrains nearby bubbles in the flow pattern. The same logic would apply when two bubbles of equal size are adjacent to each other on a warm wall. Each bubble drives thermocapillary flow and hence entrains the other in its flow pattern, which drives the aggregation. Our objective here is to perform experiments where the temperature gradient at the wall is well known and controlled. The theory can be quantitatively tested by studying aggregation of bubble pairs of equal size, and by varying system parameters such as temperature gradient, bubble size and fluid viscosity. The results are then compared with the theory in a quantitatively rigorous manner. We demonstrate that the theory without adjustable parameters is capable of quantitatively modeling the rate of aggregation of two bubbles.

The equations goveming the thermocapillary flow around a single stationary bubble on a heated or cooled wall in a semi-infinite domain were solved. Both Reynolds number and Marangoni number were much less than unity. The critical result is that liquid in the vicinity of a warm wall flows toward a stationary collector bubble (Figure 1). Consequently the thermocapillary flow around the stationary bubble entrains another bubble toward itself. The bubbles undergo hindered translation parallel to the wall with velocity $\mathbf{U}$ while the fluid flow field is described with $\mathbf{u}$. Two velocities were equated by using a wall hindrance parameter $q$ :

$$
\mathbf{U}=q \mathbf{u}
$$

which shows the velocity of bubble is proportional to the entraining velocity. The hindrance parameter $q$ can experimentally be measured independently. $q$ can also be calculated by solving the equations of motion for a bubble translating parallel to a solid wall.

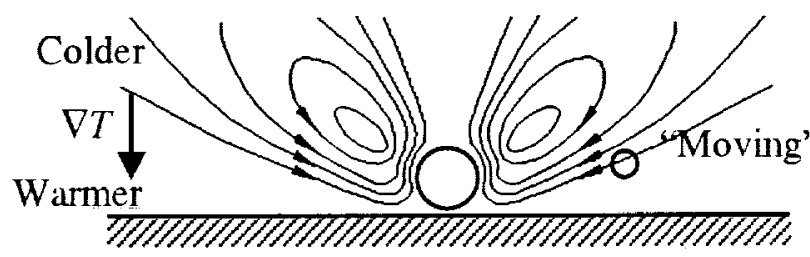

Figure 1 Streamlines of the flow around a bubble near a heated wall. 
The experimental cell is cylindrical with an ID of $10 \mathrm{~cm}$ and consists of a $1 \mathrm{~cm}$ deep main cell filled with silicone oil and flanked by two thermal reservoirs. The upper thermal reservoir was heated and the lower thermal reservoir was cooled so that the bubbles aggregate. Two types of silicone oil ( $\eta=0.02$ and $0.50 \mathrm{~Pa} \mathrm{~s}$ ) were used. Two equal sized air bubbles were injected into the cell with a syringe. The center-to-center distance of bubbles was observed through a microscope. Bubble radius ranged from $0.40 \mathrm{~mm}$ to $0.65 \mathrm{~mm}$ and the temperature gradients along with the cell ranged from 1400 to $5000 \mathrm{~K} / \mathrm{m}$.

The bubbles aggregated when heat flows from the wall to the fluid. The velocities of bubbles were in the range of $1-10 \mu \mathrm{m} / \mathrm{s}$. The separation $r$ decreased more quickly when the temperature gradient was higher, bubble size was larger, and the oil viscosity was lower. $r$ decreased more rapidly as the bubbles approached each other. Figure 2 is a plot of scaled data by appropriate time scale and bubble radius. Dimensionless time was arbitrarily set to be zero when the dimensionless center-to-center distance between the bubbles was 4 . All the bubble trajectories fall onto one line, especially in the range of dimensionless distance from 4 to 3 . This means the relative movement of the bubble pair is proportional to the temperature gradient and bubble size and it is inversely proportional to the viscosity of the oil. This result strongly suggests that the thermocapillary flow-based aggregation mechanism is correct.

A value of $q$ can be estimated by fitting the scaled data to Eq. [1]. A best fit value of $q$ was obtained as $q=0.26$ with a standard deviation of 0.03 . Independent experimental results for $q$ for a $0.5 \mathrm{~mm}$ radius bubble, give values of $q$ in the range 0.11 to 0.23 . The value of $q$ obtained from solving the equations of motion reveals $q$ has values in the range $0.23-0.30$. Since the full scale of possible values of $q$ is zero to one, the maximum deviation of independently determined values of $q$ from the best fit value was $15 \%$ of this full scale. Thus reasonable quantitative agreement between theory and experiment has been obtained.

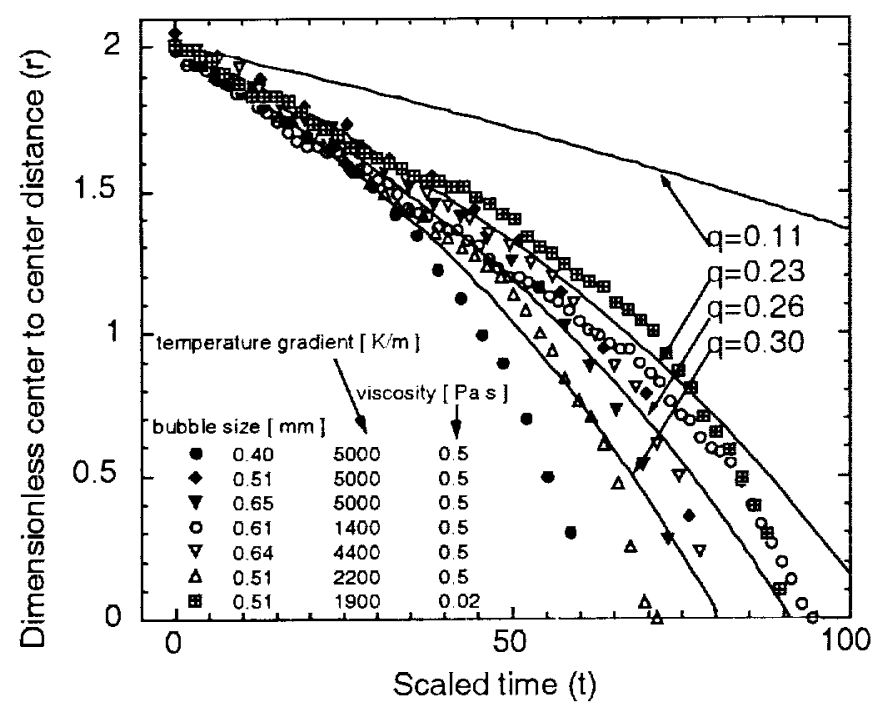

This work was supported by the NASA Microgravity Program, grant NAG3-2159. We acknowledge the use of a macro for the image software written by Professor Darrell Velegol of the Pennsylvania State University.

\section{REFERENCES}

[1]. Sides, P. J., Ph.D. Thesis, University of California, Berkeley, California (1981).

[2]. Sides, P. J., and Tobias, C. W., J. Electrochem. Soc., 132, 583 (1985).

[3]. Guelcher, S. A., Solomentsev, Y. E., Sides, P. J., and Anderson, J. L., J. Electrochem. Soc., 145(6), 1848 (1998).

Figure 2 Scaled experimental bubble trajectories trajectory. The ordinate shows the center-tocenter distance of the bubbles scaled by the bubble radius, and the abscissa shows the dimensionless time. Solid lines are the bubble trajectory calculated from Eq. 1. 


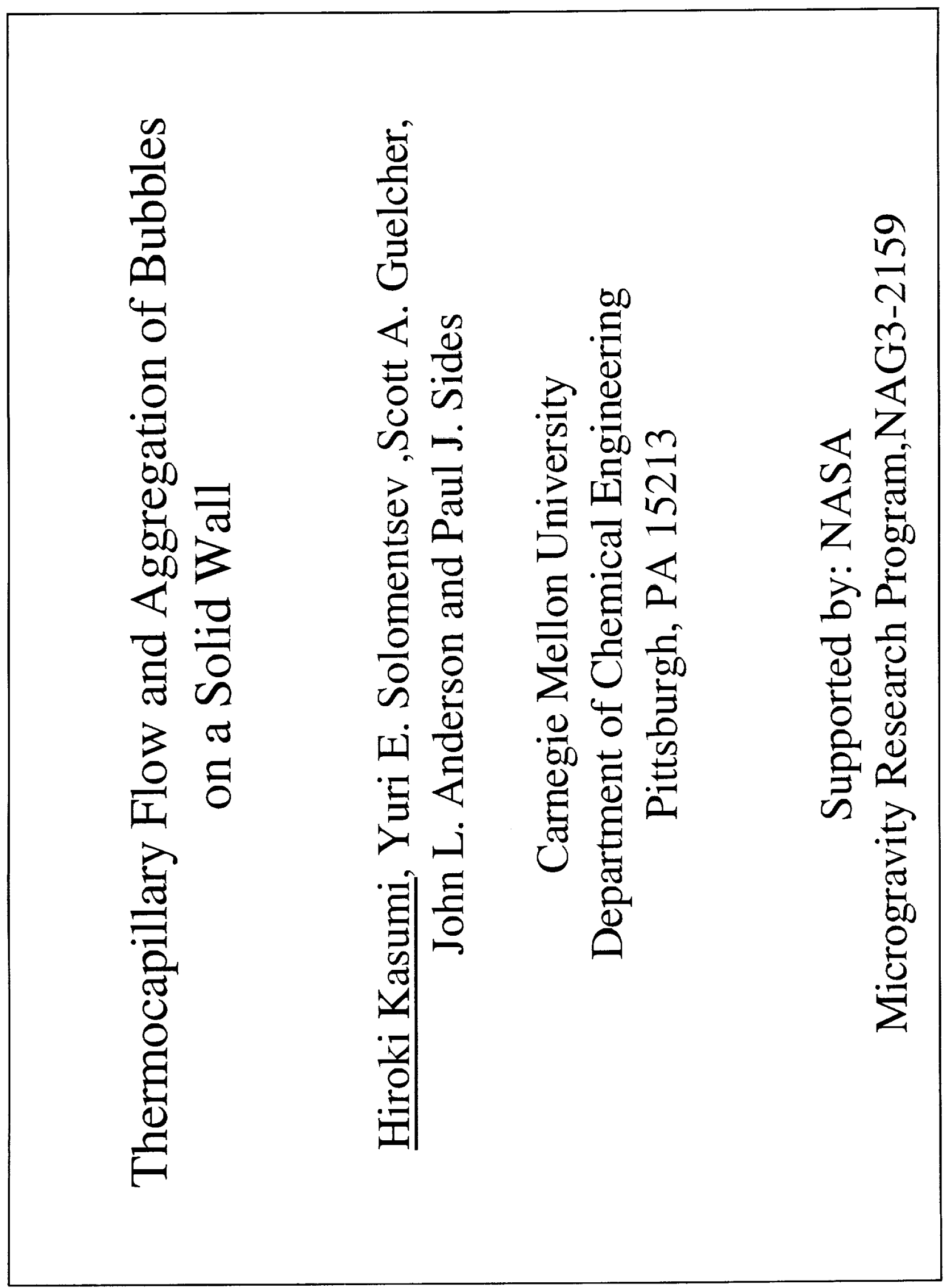




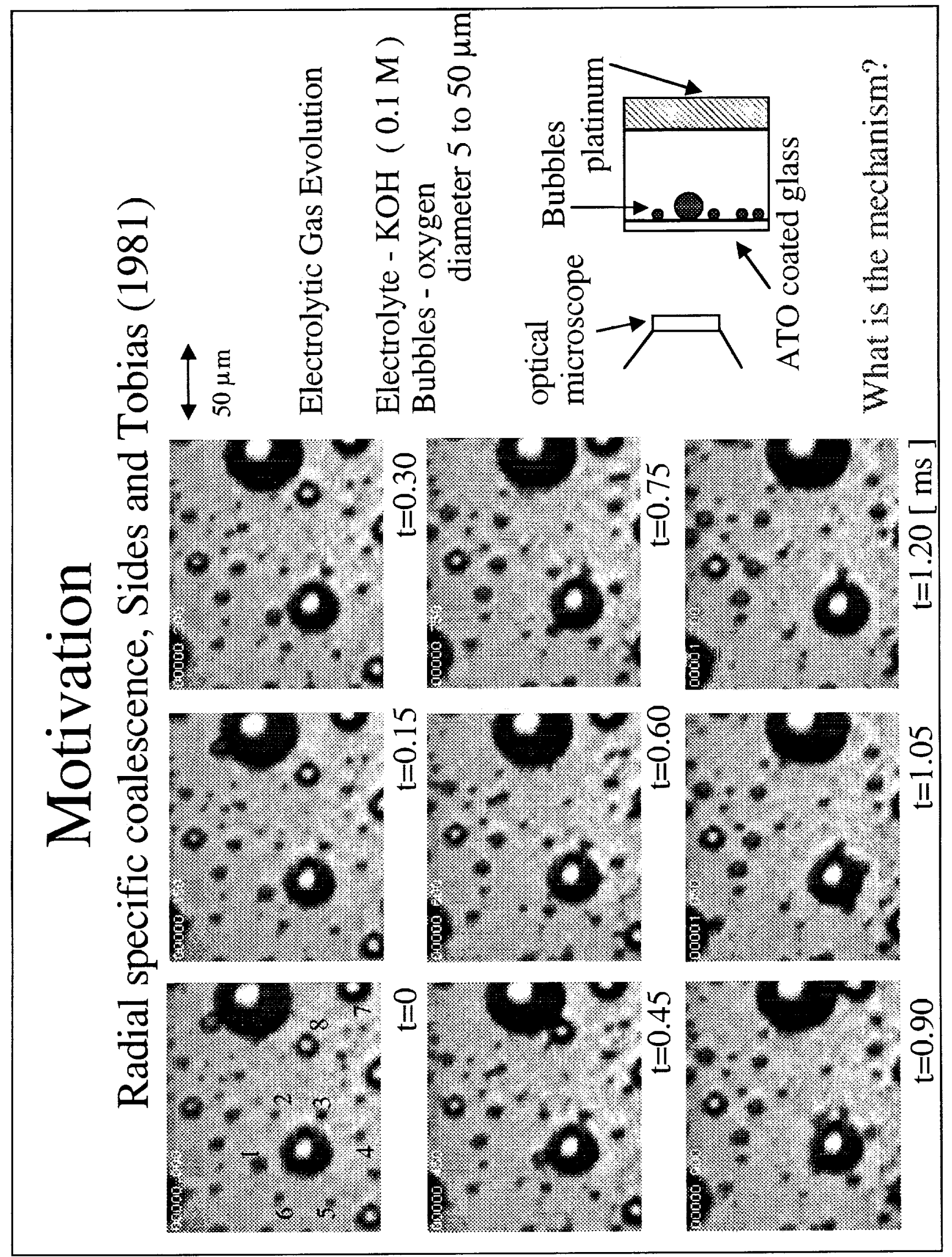




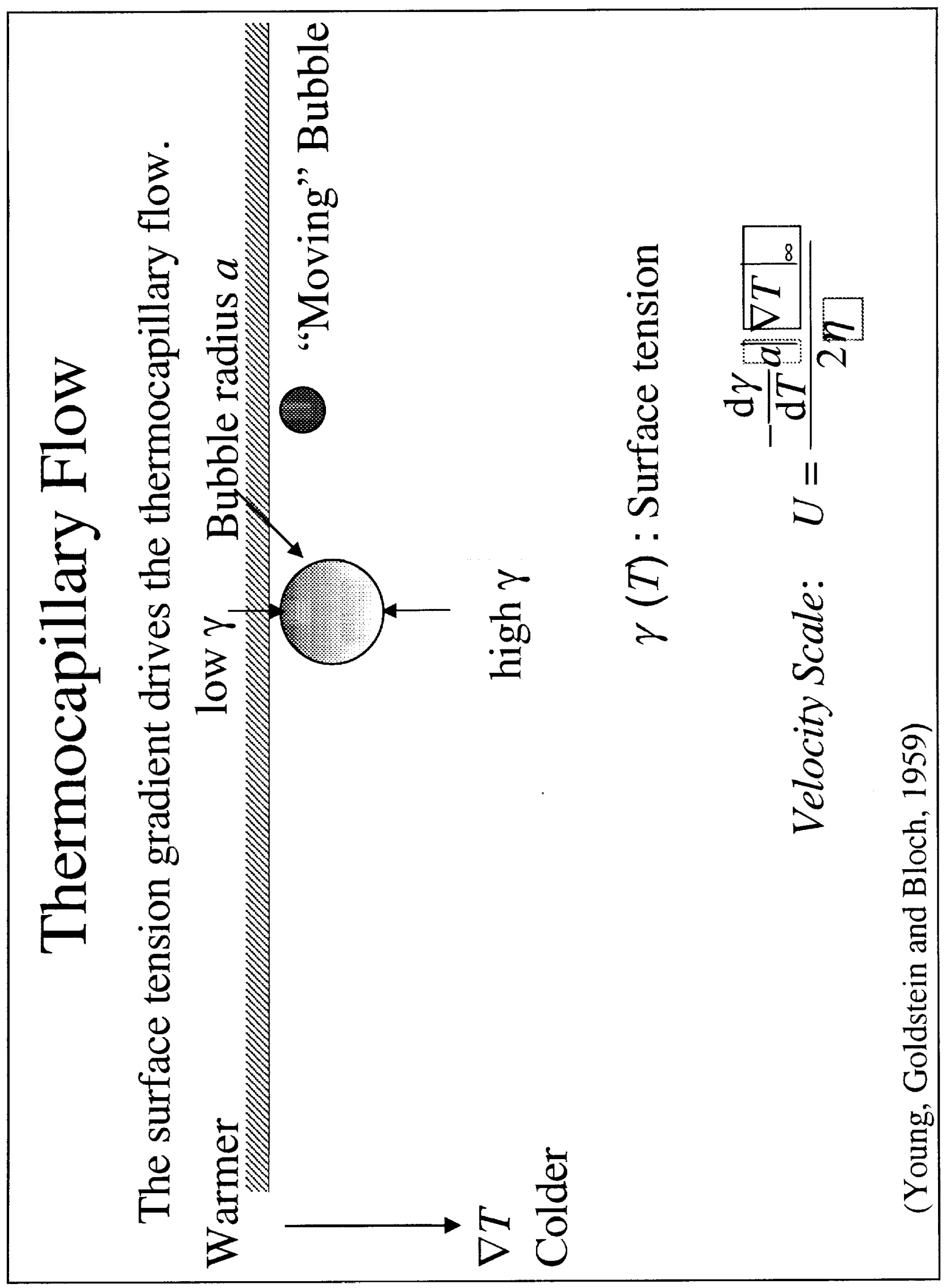



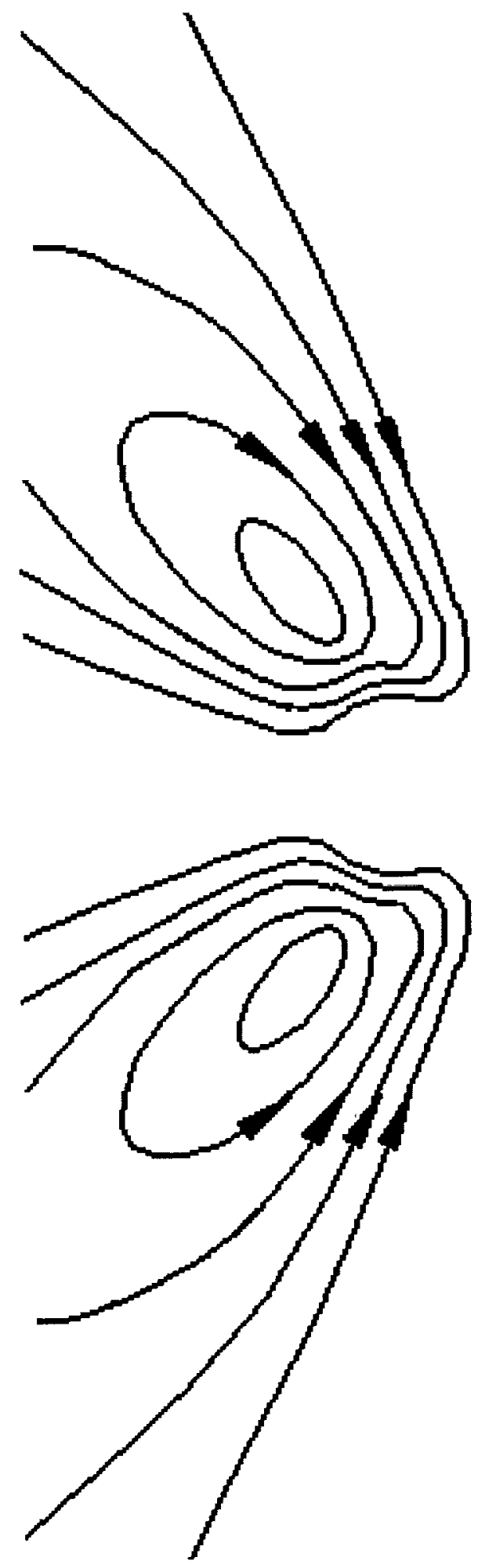


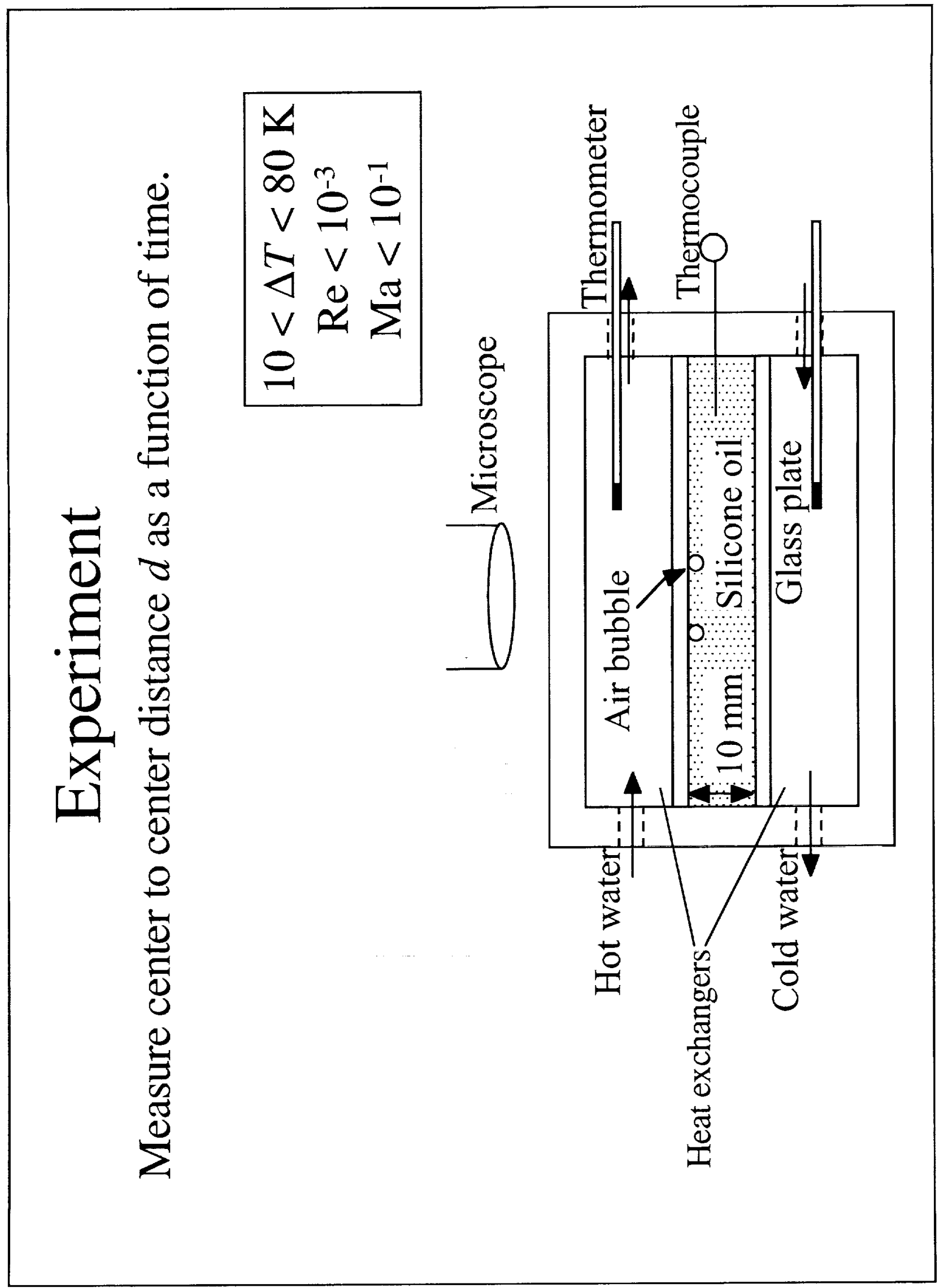




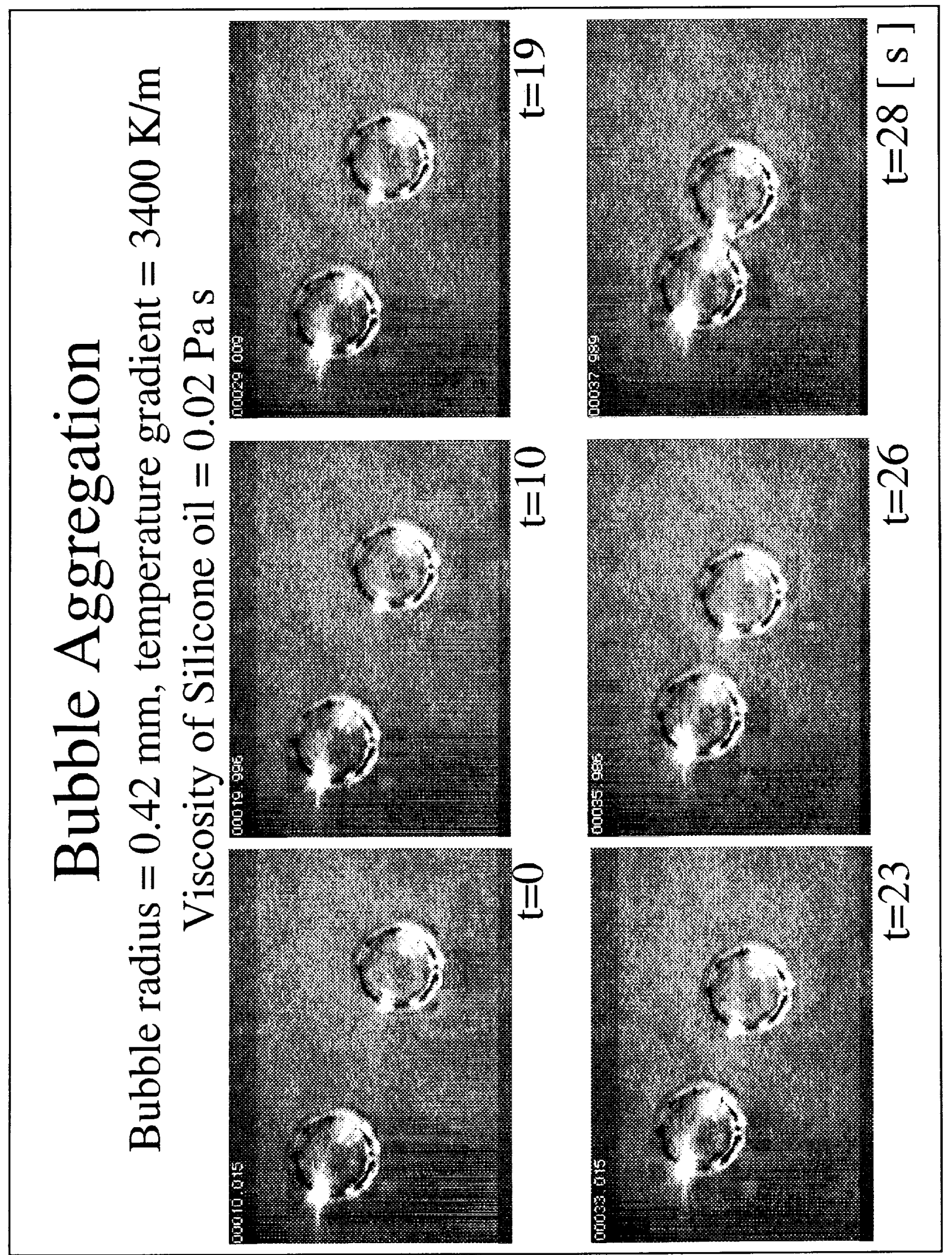




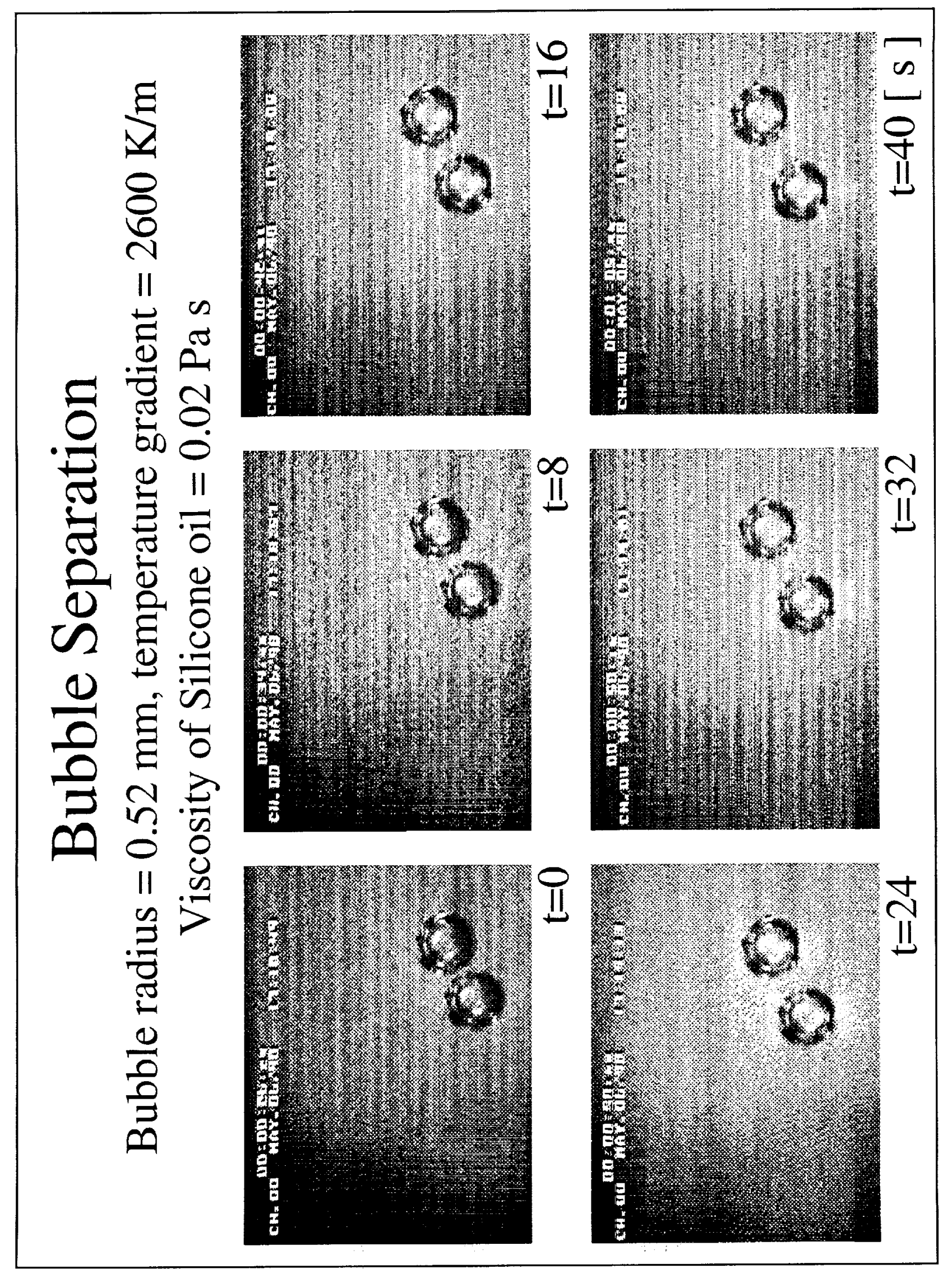




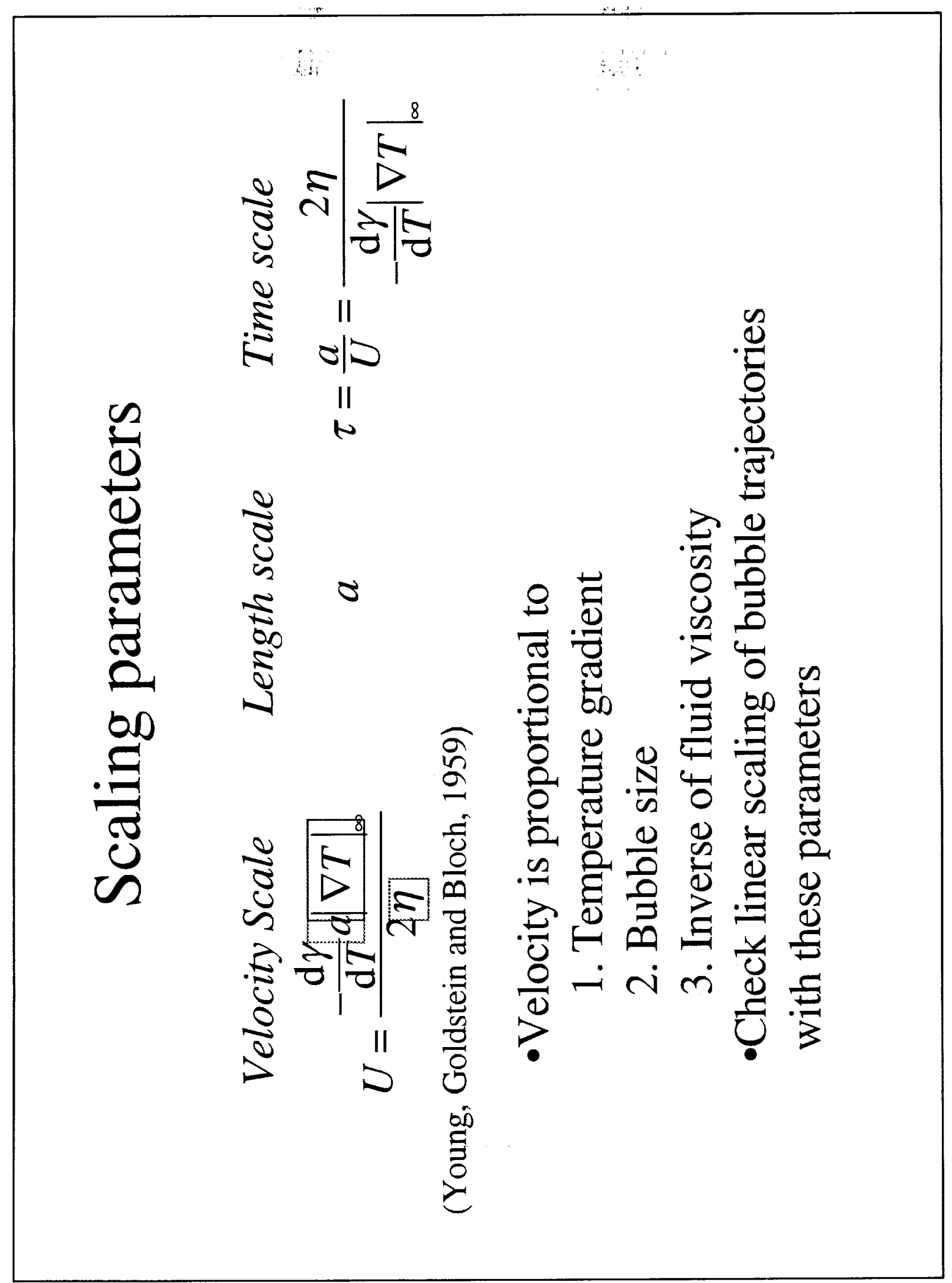




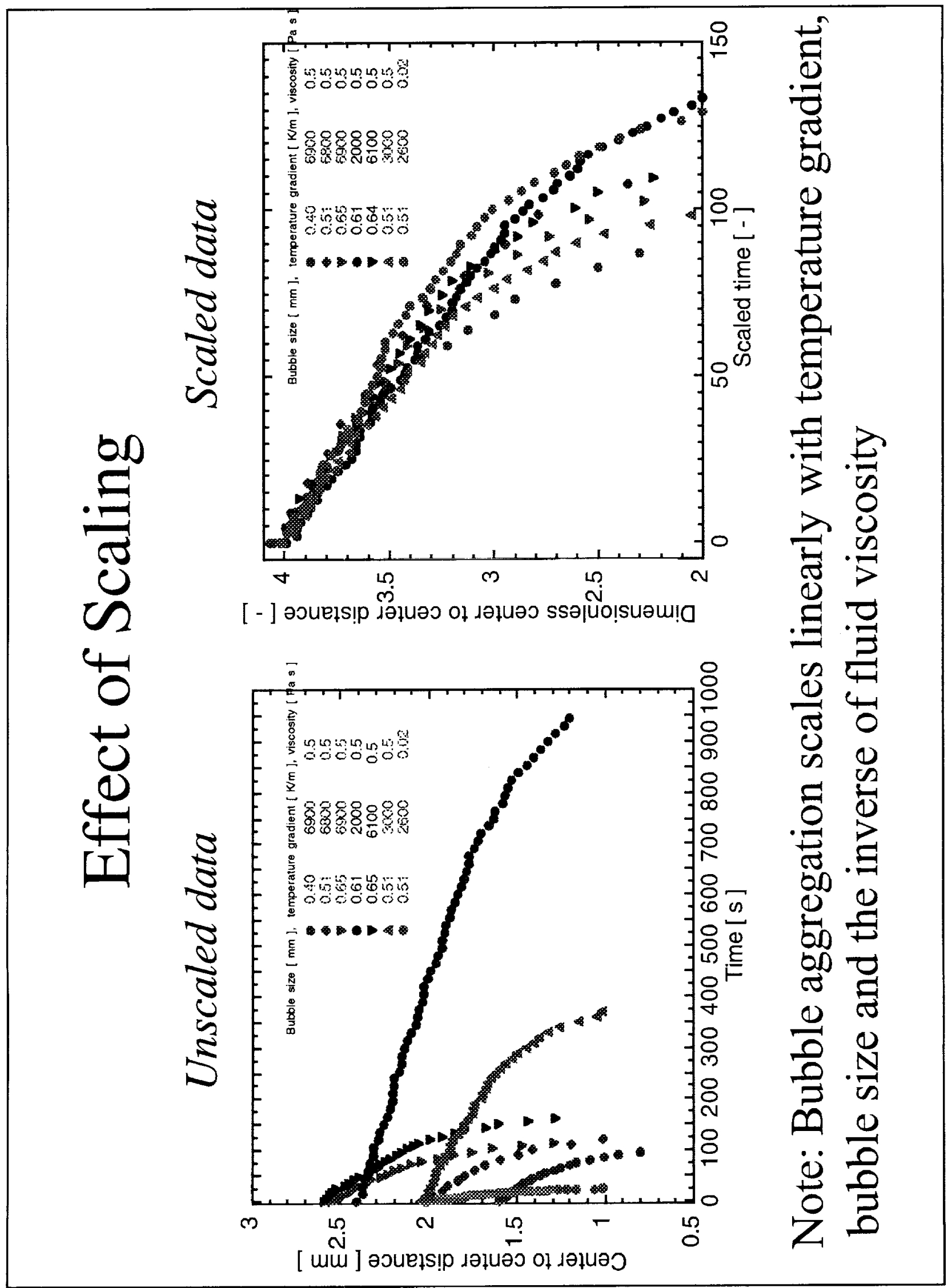




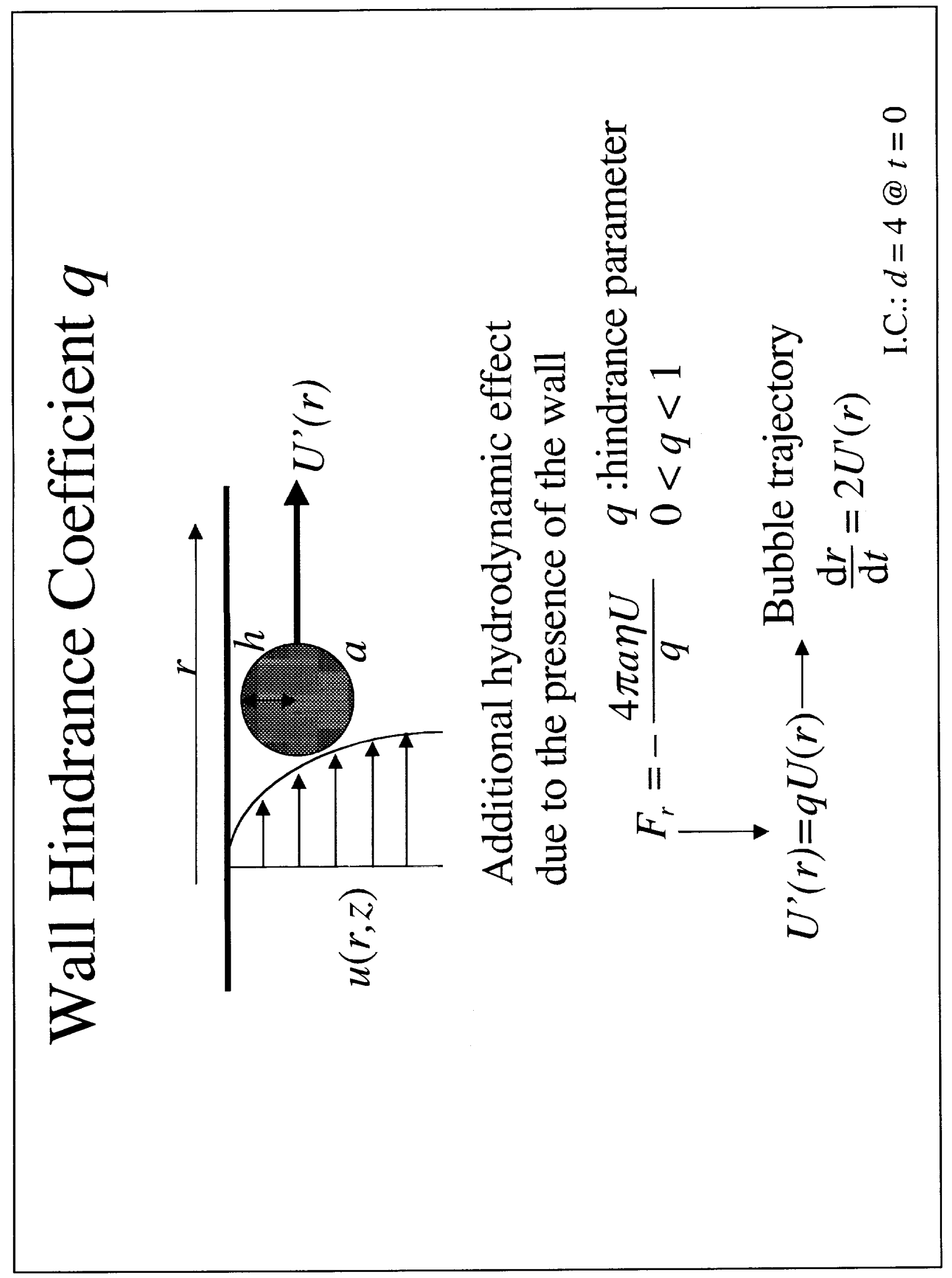




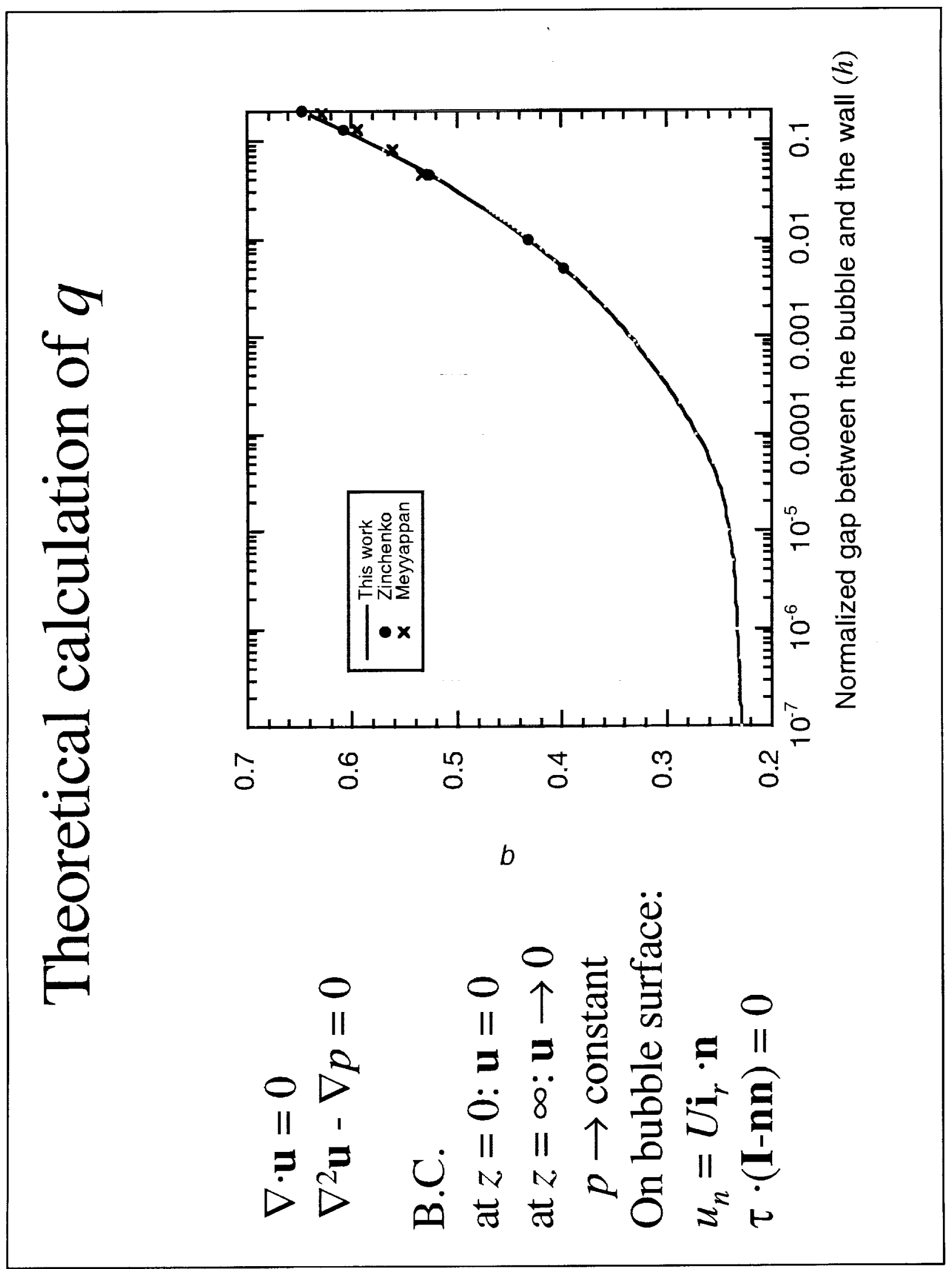




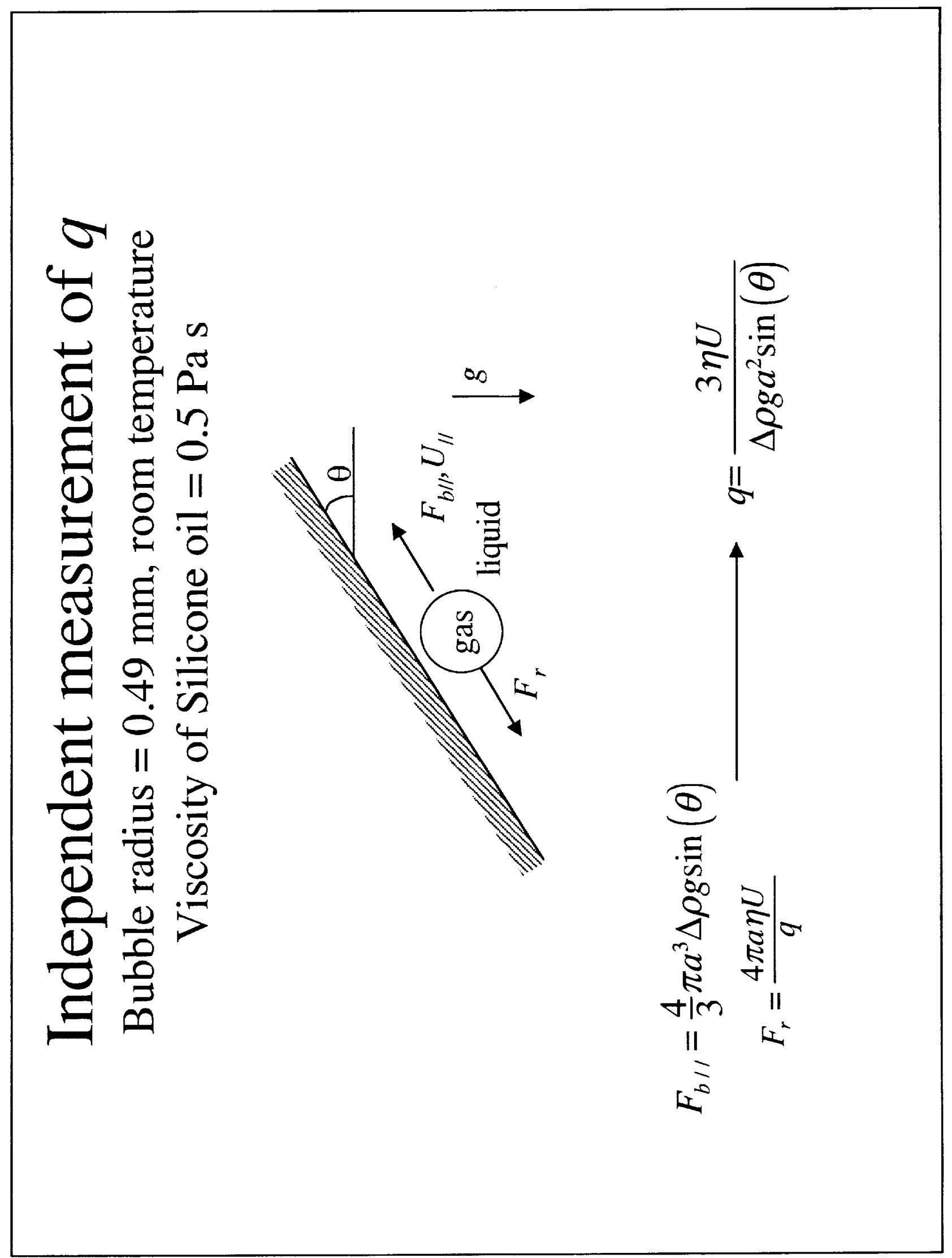




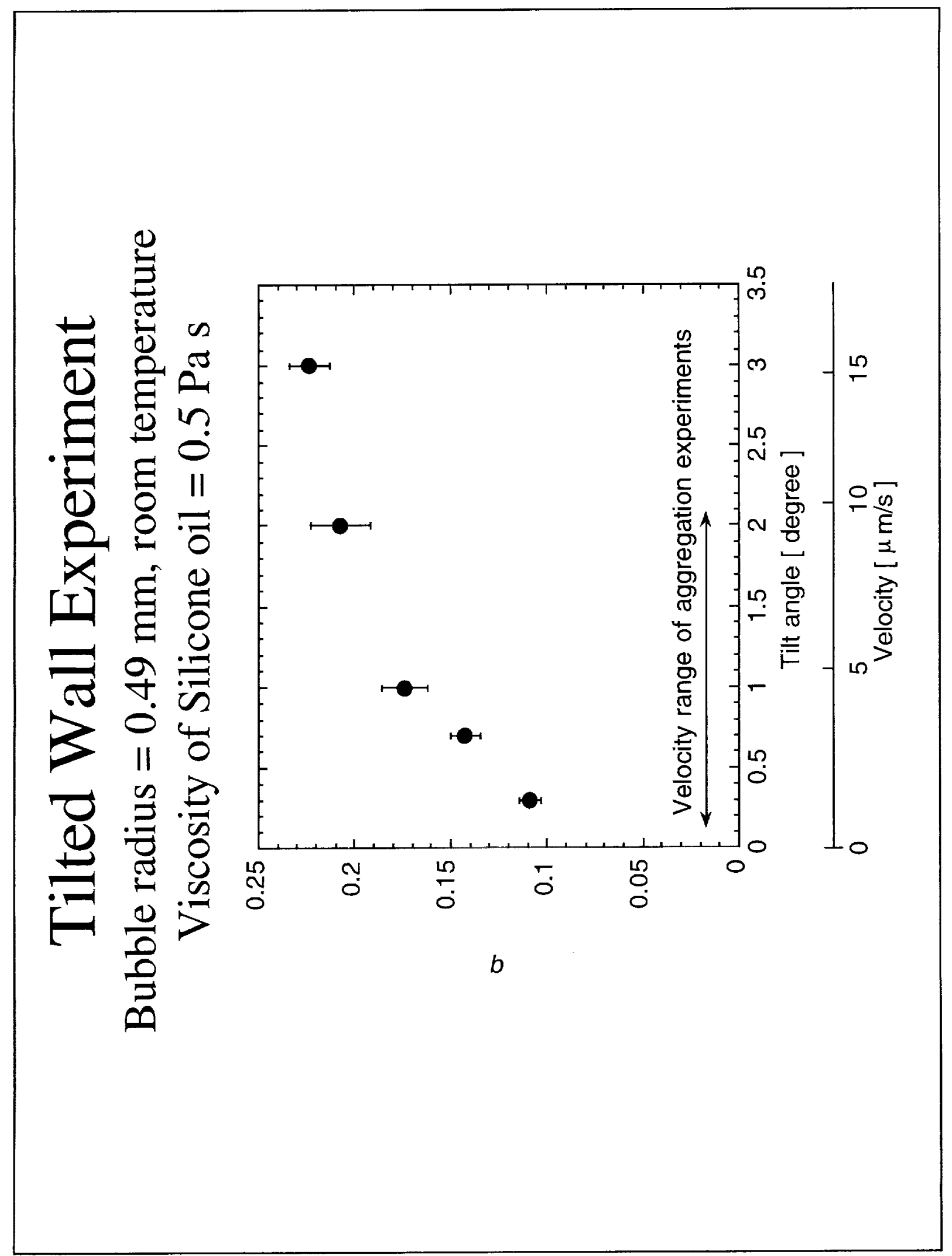




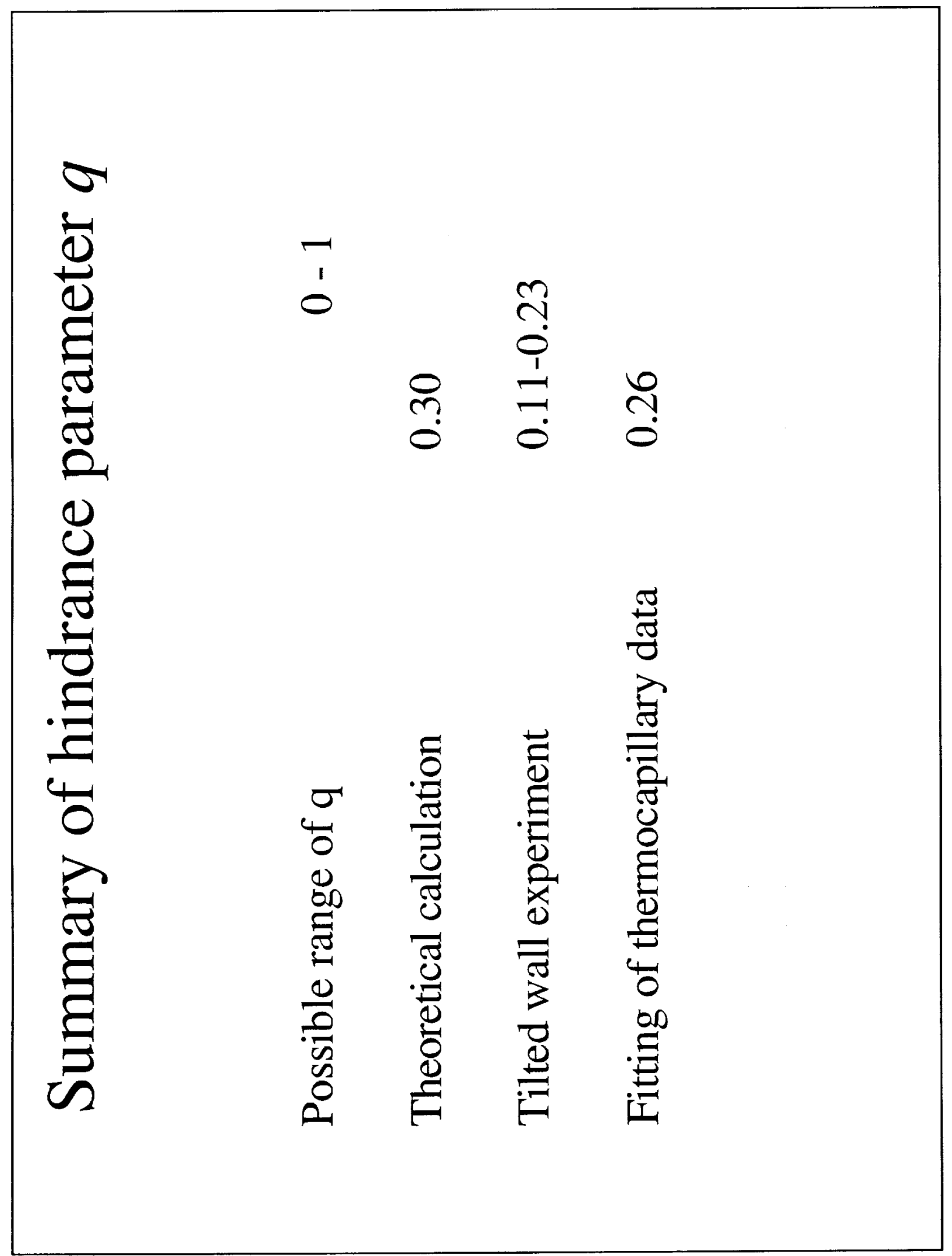




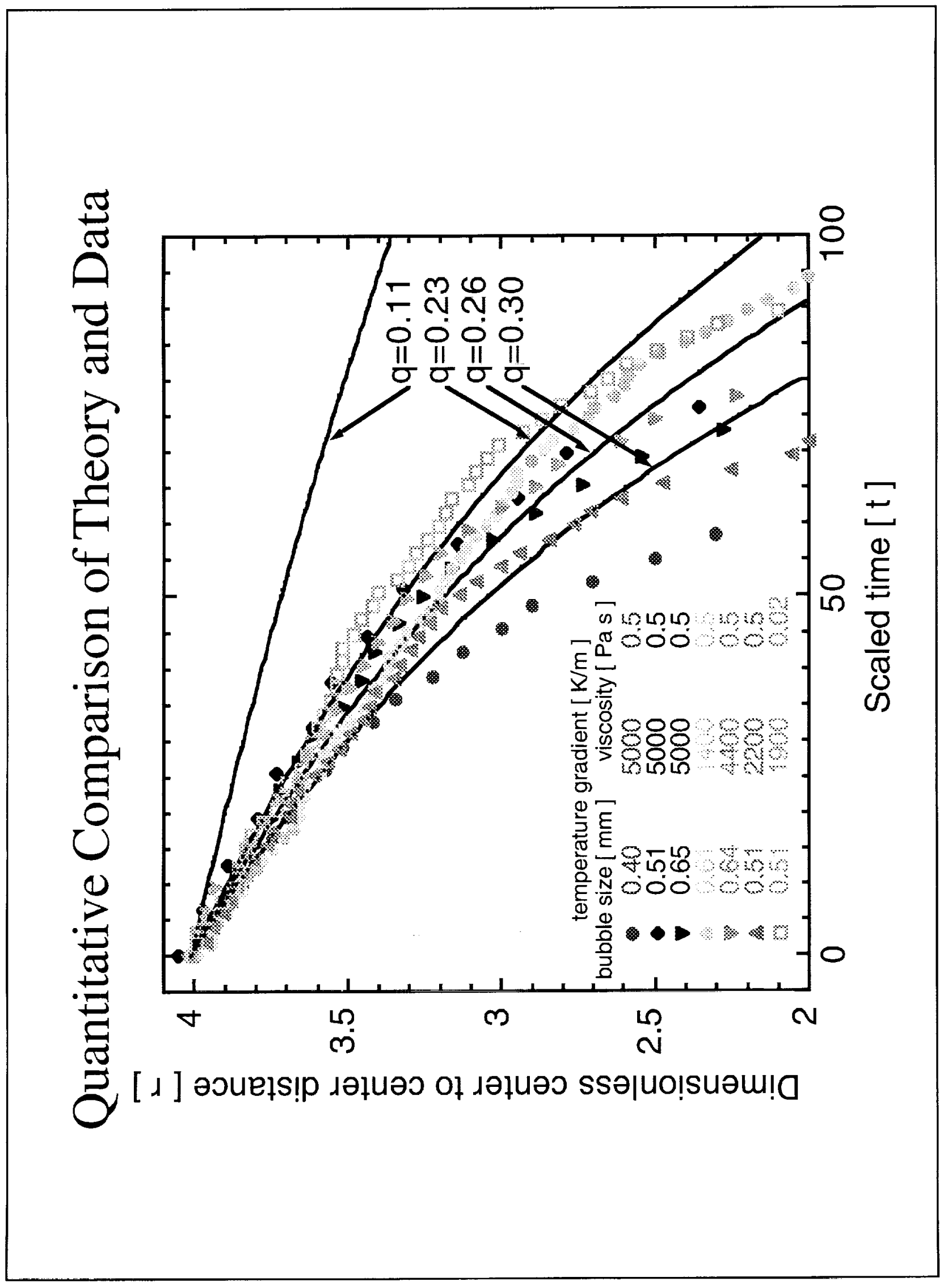




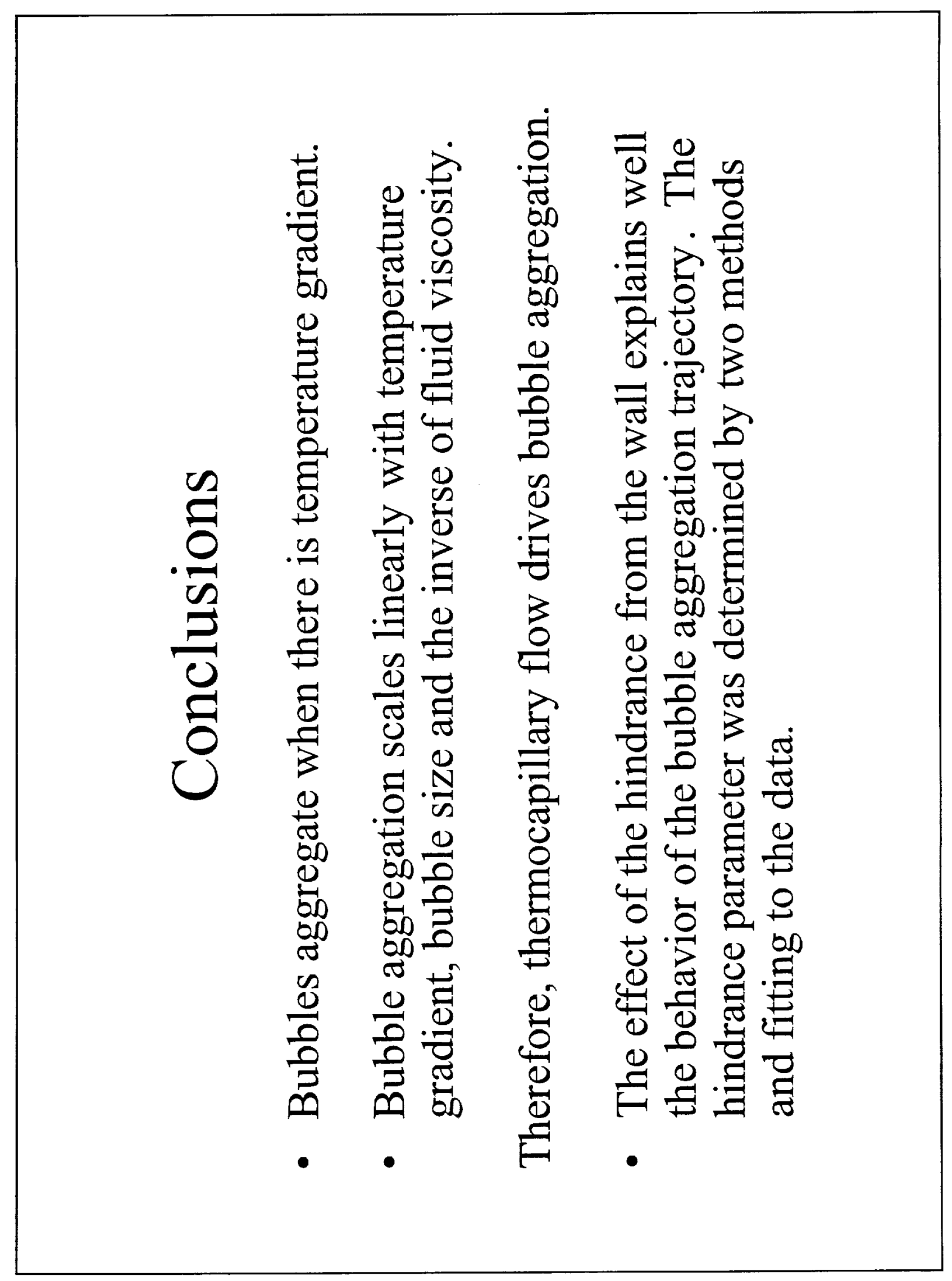

